\section{SCIENCE CHINA \\ Physics, Mechanics \& Astronomy}

\title{
Harvesting transverse thermoelectricity in topological semimetals
}

\author{
XianHui Chen ${ }^{*}$ \\ Department of Physics, University of Science and Technology of China, Hefei 230026, China
}

Received October 14, 2019; accepted October 18, 2019; published online November 19, 2019

\begin{tabular}{ll}
\hline Citation: & X. H. Chen, Harvesting transverse thermoelectricity in topological semimetals, Sci. China-Phys. Mech. Astron. 63, 237031 (2020), https://doi.org/ \\
10.1007/s11433-019-1465-8
\end{tabular}

The thermoelectric effect refers to the phenomenon in which an electric voltage is directly generated from a temperature difference or vice versa. The two primary such effects are the Seebeck and the Peltier effects discovered in the early nineteenth century [1]. These two effects provide the basis for functional thermoelectric devices, and are used to harvest electrical energy from waste heat or as solid-state Peltier cooler, see Figure 1(a). Here, the most challenging issue is to increase the thermoelectric figure of merit $z T$, which is determined by several interrelated material parameters including the Seebeck coefficient, the electrical and thermal conductivities, as well as the working temperature.

The aforementioned Seebeck and Peltier effects are known as longitudinal thermoelectric effects because the electrical and thermal fluxes are parallel to each other. Considering a tensor description of the thermoelectric transport, their transverse counterparts can be defined as well. Actually, the Nernst effect, i.e., the transverse electrical field generated by a longitudinal temperature gradient (or reversely, the Ettingshausen effect) in the presence of magnetic field has long been known since its discovery in the late nineteenth century in the semimetal bismuth [2]. In the following century, however, Nernst effect has remained largely unexplored because it is generally much too small compared to its longitudinal counterpart. Nowadays, we have learned that bismuth shows relatively large Nernst effect due to its small Fermi surface, the large carrier mobility and the electronhole compensation [3]. In particular, as shown in Figure 1(b),

*Corresponding author (email: chenxh@ustc.edu.cn)
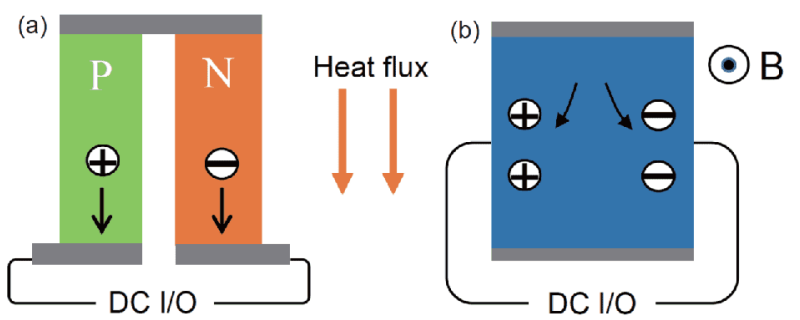

Figure 1 (Color online) Schematics of longitudinal (a) and transverse (b) thermoelectric modules. While $n$ and $\mathrm{p}$ type materials are needed for the longitudinal thermoelectric module, one material with compensated electrons and holes suffices for the transverse case. When a DC electrical power input is provided, they work as Peltier or Ettingshausen cooler; when a temperature difference is applied, DC electrical power can be harvested either longitudinally (a) or transversely (b).

the Nernst response of electrons and holes can add to each other, leading to an enhanced transverse thermoelectric effect by bipolar transport [4,5]. In other words, electrons and holes contribute equally and additively in a transverse thermoelectric configuration (Figure 1(b)), in sharp contrast to the longitudinal thermoelectric effect, where coexistence of electrons and holes will diminish the Seebeck effect.

A recent paper of Xiang et al. [6] published in SCIENCE CHINA Physics, Mechanics \& Astronomy has demonstrated that the transverse thermoelectricity of topological semimetals is promising for thermoelectric application. By studying the Nernst effect in the prototypical Dirac semimetal $\mathrm{Cd}_{3} \mathrm{As}_{2}$, they can define a transverse figure of merit that amounts to 0.5 at $2 \mathrm{~T}$ at room temperature. This value is 
even larger than the enhanced longitudinal thermoelectric figure of merit obtained at the same field and temperature [7]. Interestingly, such transverse thermoelectric effect has been shown to be easily optimized by tuning the Fermi level towards the Dirac/Weyl nodes. In view of the band structure characteristics, obviously, topological semimetals fulfill all the conventional requirements for large Nernst effect, as has been argued for bismuth. More importantly and excitingly, topological semimetals can have additional transverse thermoelectric effect, known as anomalous Nernst effect that arises from the pronounced Berry curvature near the Fermi level [8].

Just like the much larger Hall conductivity over its longitudinal counterpart that is generically observed in many topological semimetals in small fields, the transverse thermoelectric effects can largely surmount their longitudinal counterparts. Due to the large carrier mobility, the magnetic field needed for a dominating transverse thermoelectricity can be reduced to be as small as below $1 \mathrm{~T}$. Moreover, due to the topologically protected band structure, topological semimetals can in principle reveal high carrier mobility and low thermal conductivity simultaneously due to, e.g., heavy constituent elements and intrinsic lattice defects as realized in $\mathrm{Cd}_{3} \mathrm{As}_{2}$, a Cd-deficient of the ideal $\mathrm{Cd}_{4} \mathrm{As}_{2}$ formula.
Other merits of transverse thermoelectric effects over their longitudinal counterparts include, for example, that one does not need both $\mathrm{n}$ and $\mathrm{p}$ types of thermoelectric materials for constructing one device. Because the electrical and thermal currents are orthogonal and decoupled in the transverse case, a high electrical conductivity and a low thermal conductivity desired for large transverse figure of merit can in principle be easily realized. In-depth investigations on various topological semimetals along this line appear to be highly interesting.

1 H. J. Goldsmid, Introduction to Thermoelectricity (Springer, Berlin, Heidelberg, 2009).

2 A. V. Ettingshausen, and W. Nernst, Ann. Phys. Chem. 265, 343 (1886).

3 K. Behnia, and H. Aubin, Rep. Prog. Phys. 79, 046502 (2016), arXiv: 1601.06647.

4 R. Bel, K. Behnia, and H. Berger, Phys. Rev. Lett. 91, 066602 (2003).

5 J. L. Cohn, B. D. White, C. A. M. dos Santos, and J. J. Neumeier, Phys. Rev. Lett. 108, 056604 (2012), arXiv: 1201.2154.

6 J. S. Xiang, S. L. Hu, M. Lyu, W. L. Zhu, C. Y. Ma, Z. Y. Chen, F. Steglich, G. F. Chen, and P. J. Sun, Sci. China-Phys. Mech. Astron. 3, 237011 (2020), arXiv: 1908.00184.

7 H. Wang, X. Luo, W. Chen, N. Wang, B. Lei, F. Meng, C. Shang, L. Ma, T. Wu, X. Dai, Z. Wang, and X. Chen, Sci. Bull. 63, 411 (2018).

8 T. Liang, J. Lin, Q. Gibson, T. Gao, M. Hirschberger, M. Liu, R. J. Cava, and N. P. Ong, Phys. Rev. Lett. 118, 136601 (2017), arXiv: 1610.02459 . 